\title{
Closed Traumatic Avulsion of Extensor Digitorium Communis and Extensor Indicis Proprius of Index Finger at the Musculotendinous Junction
}

\author{
Sefeane $\mathrm{T}^{*}$, Aden AA and Mshuqwana $\mathrm{P}$
}

The Hand Unit, Chris Hani Baragwanath Hospital, Department of Orthopaedic Surgery, University of the Witwatersrand, Johannesburg, South Africa

*Corresponding author: Sefeane T, The Hand Unit, Chris Hani Baragwanath Hospital, Department of Orthopaedic Surgery, University of the Witwatersrand, Johannesburg, South Africa, E-mail: tatolos@me.com

Citation: Sefeane T, Aden AA, Mshuqwana P (2016) Closed Traumatic Avulsion of Extensor Digitorium Communis and Extensor Indicis Proprius of Index Finger at the Musculotendinous Junction. J Case Rep Stud 4(1): 102. doi: 10.15744/2348-9820.4.102. doi: 10.15744/2348-9820.4.102

Received Date: October 28, 2015 Accepted Date: February 11, 2016 Published Date: February 15, 2016

\begin{abstract}
Introduction: Closed traumatic rupture of extensor tendons of the fingers at the musculotendinous is rare. In fact the common sites of a closed rupture are distal attachment at the distal phalanx as well as at the attachment of the central slip at the base of the middle phalanx. The diagnosis of these injuries at the latter sites is straightforward.

Case presentation: We present a 19 year-old right hand dominant motor mechanic male patient who sustained injury of his left forearm while swinging from gymnastic bars. He reports that while swinging, he felt a violent snap at the dorsum of his left forearm while his body swung down from a height. He thinks that the grip of the left hand did not release in time following the downward swing of his body. He presented few days after the injury complaining of pain, swelling and inability to actively extend the index finger. On surgical exploration, it was documented that both the extensor digitorium of the index as well as the extensor indicis proprius were torn at their musculotendinous junctions. Both tendons were sutured side to side before the resultant joined tendon was sutured to the extensor tendon of the middle finger. A below-elbow volar slab was applied for 6 weeks followed by hand therapy. The patient was seen for some time and came back only once for follow-up. At 8 months postoperatively, he was back to his work with full recovery demonstrating isolated index finger extension.

Discussion: Closed traumatic rupture of the extensor tendons at the musculotendinous junction is very rare. This case serves to highlight, the peculiar mechanism of injury; the violent snap that results from the rupture and the presented clinical picture. Like this case, if surgery is done in time, a good outcome is to be expected when side-to-side suturing of the two tendons is done and the resultant joined tendon is sutured to the adjacent middle finger extensor tendon.
\end{abstract}

Keywords: Extensors; Index finger; Rupture; Musculotendinous junction; Index finger

\section{Introduction}

Closed traumatic rupture of tendons at the musculotendinous junction occurs when there is a sudden longitudinal pull of the tendon against a contracting muscle as in resisted extension or flexion of a finger [1]. The weakest point in the whole musculotendinous unit is however at the distal insertion point of the tendon into bone as described by Boyes [2]. When considering the extensor tendons in isolation, the most closed ruptures occur at the insertion of the tendon on the distal phalanx or the attachment of the central slip at the base of the middle phalanx.

These injuries are extremely rare and only a few have been reported in the literature (Stuart and Briggs 1993 [3], Takami 1995 [4], Mudgal 2007 [5], Sunagawa 2009 [6], and Kumora 2010 [7] with varying reported mechanism of injury. The consensus however is a mechanism of a hyperflexion force exerted suddenly on the index finger.

\section{Case Report}

We present a 22 year old motor mechanic who is also an amateur gymnast who reported injuring his forearm while swinging from a gymnastic high bar four days prior to consultation. He described feeling a violent tearing pain at the dorsum of the forearm as he released from the high bar as his body swung down. He believes that there was probably a momentary delay of release of the left hand while his body was already accelerating down. He could not continue his routine as he was in excruciating pain. He initially thought it was a torn muscle which would naturally improve in a couple of days but on day 3 he realized that there was something wrong with his left index finger which could not extend. 
At presentation he could not extend his index finger and there was a tender soft mobile mass at about the proximal end of the extensor retinaculum (Figure 1).

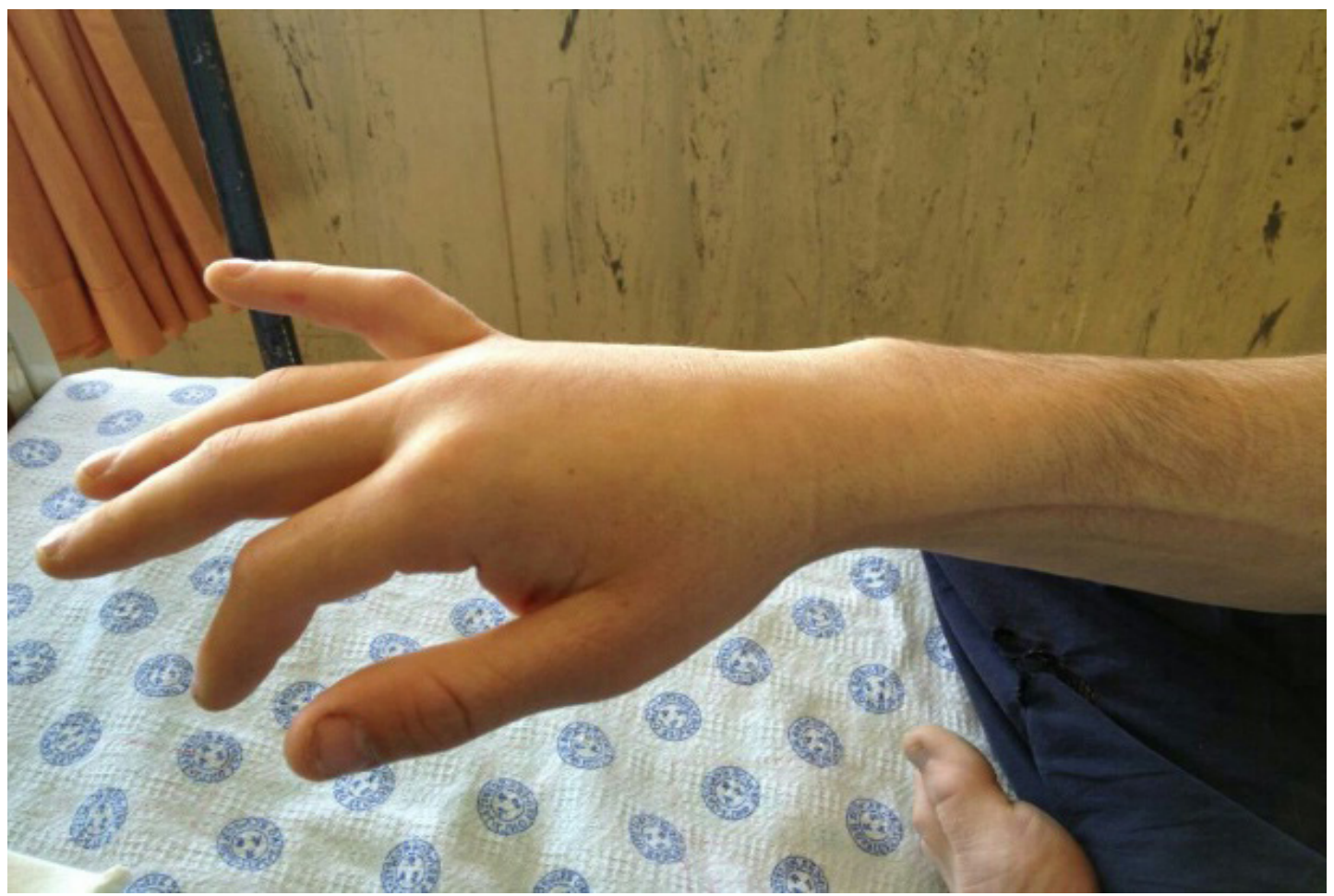

Figure 1: Clinical presentation showing the inability to extend the index finger and the dorsal swelling of the wrist

A diagnosis of rupture of both tendons was reached and an exploration and repair was scheduled. It was decided that our incision should include the swelling that was obvious at the extensor retinaculum. A longitudinal long incision was made and dissection revealed both index finger extensors ruptured at the musculotendinous junction (Figure 2,3).

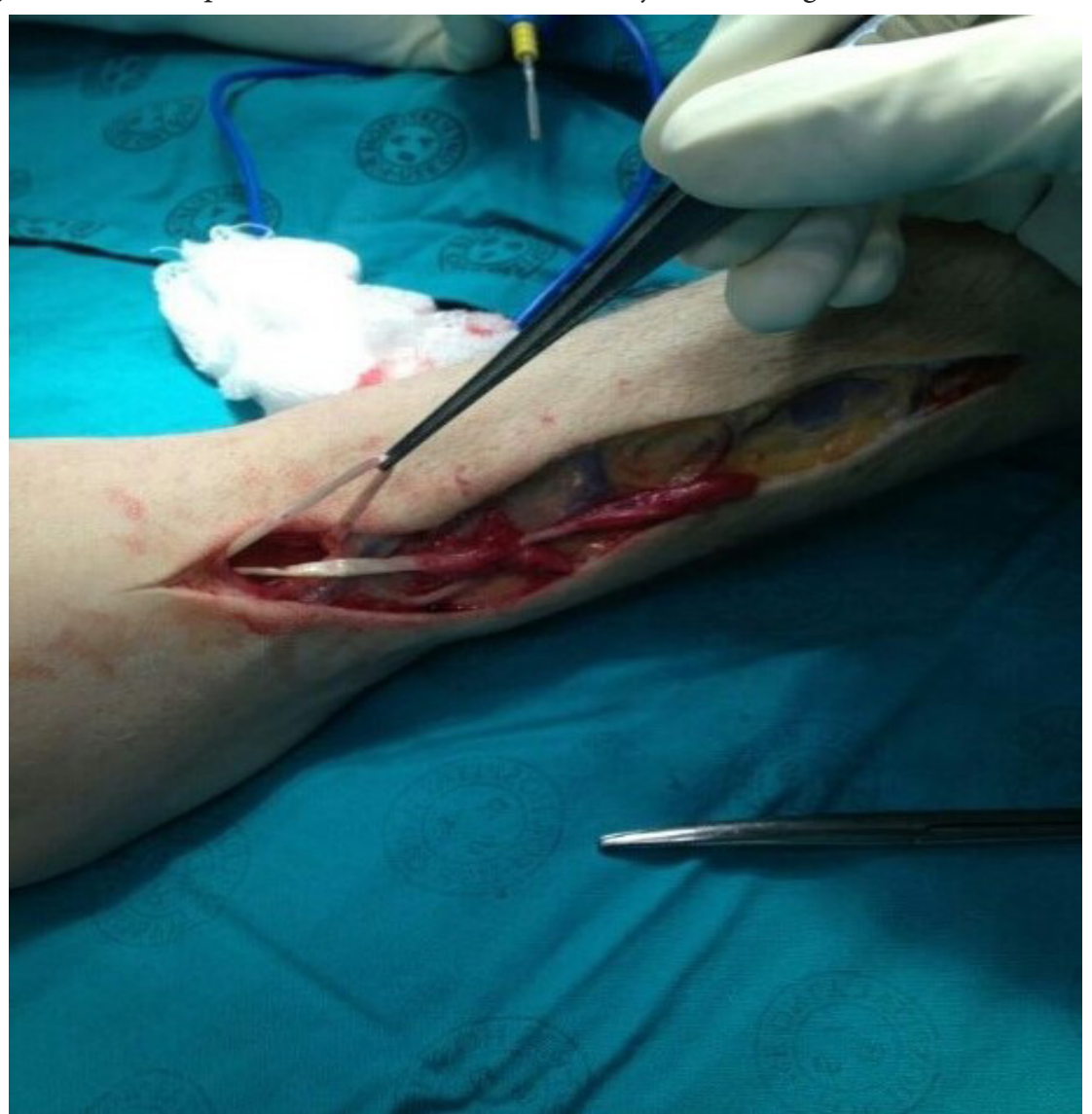

Figure 2: The Extensor Indices Proprius showing rupture at the Musculotendinous junction and the Extensor digitorium communis being pulled from the retinaculum 


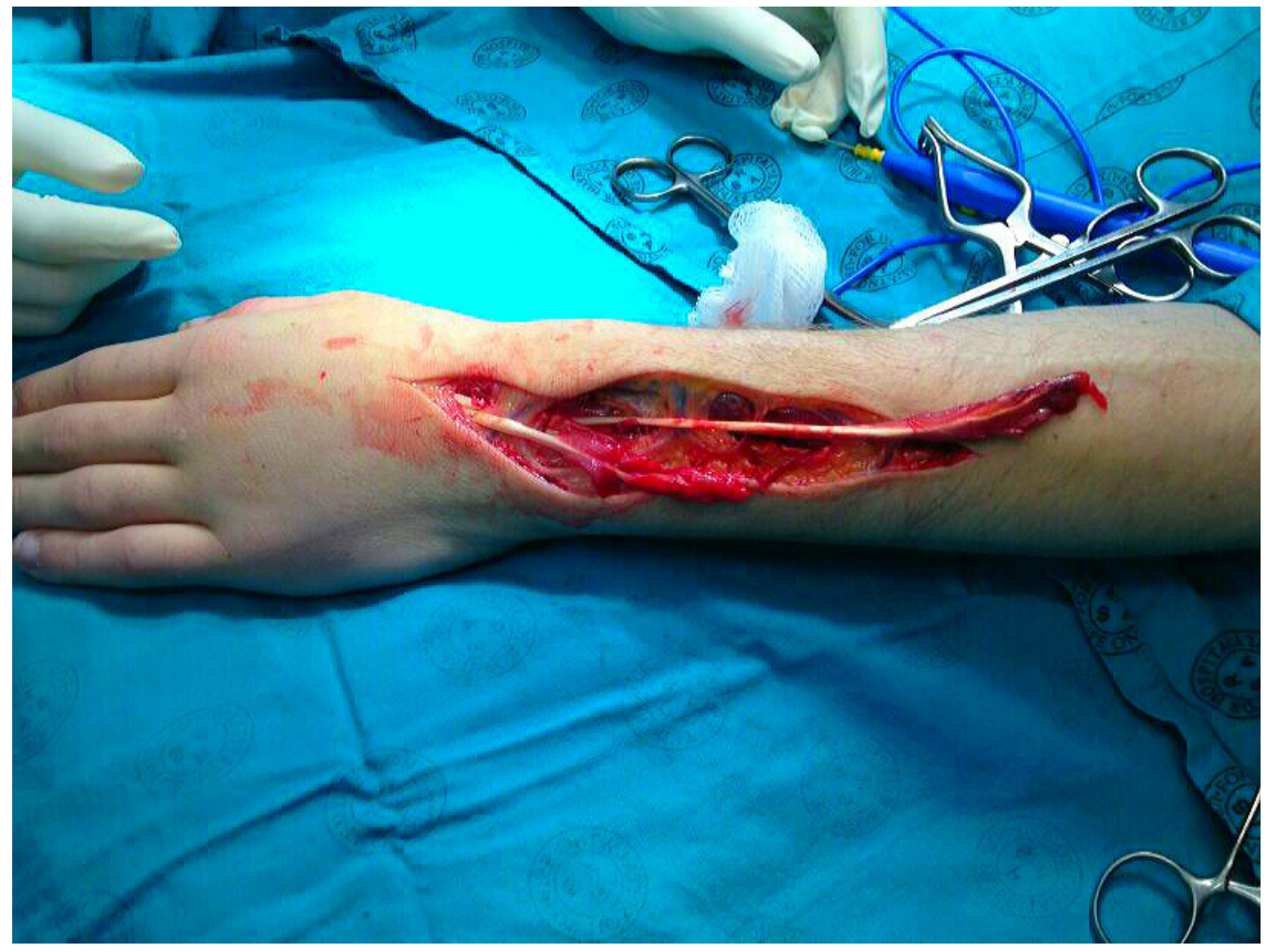

Figure 3: The two tendons after dissection showing rupture at the musculotendinous junction

The distal part of the EDC had retracted and bunched up under the extensor retinaculum forming the soft, tender and mobile mass we elicited during the clinical examination. Due to the location of the rupture, direct repair was not feasible as there was shortening and the remnant muscle belies did not look viable which would have necessitated debridement. The only viable option we had was tendon transfers. We opted to suture both tendons together and then transfer the resultant tendon to the Extensor digitorium communis of the middle finger (Figure 4).

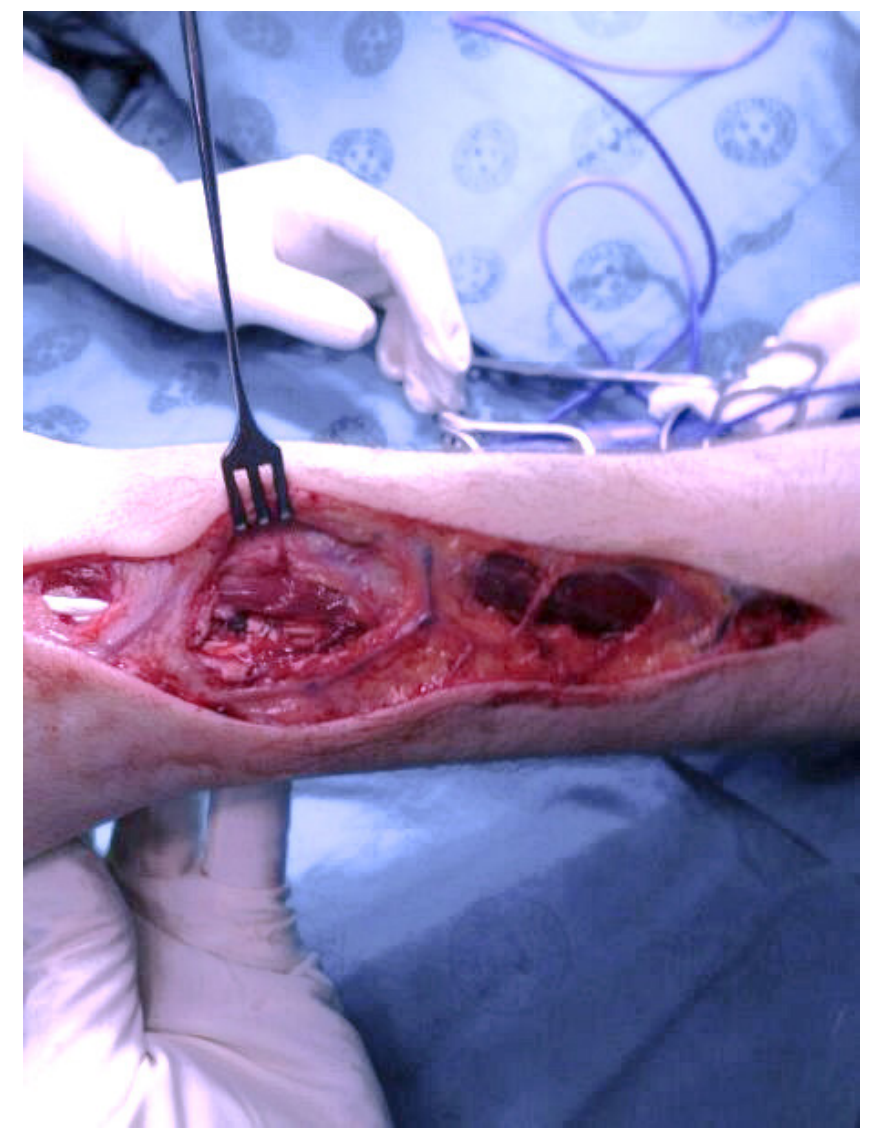

Figure 4: The two tendons were sutured together and then transferred to the Extensor digitorium communis of the middle finger 
Closure was done in layers and skin with subcuticular monocryl 5(0). A volar below elbow slab was applied to immobilize for 6 weeks after which he underwent hand therapy. We reviewed him two more times during the hand therapy routine and at final review at 8 months he was back at work and had full independent extension of the index finger (Figure 5,6). This was unexpected from our choice of tendon transfers but not unusual as we do see independent finger extension after Flexor Carpi Radialis transfer to extensor digitorium communis in Radial nerve palsy tendon transfers.

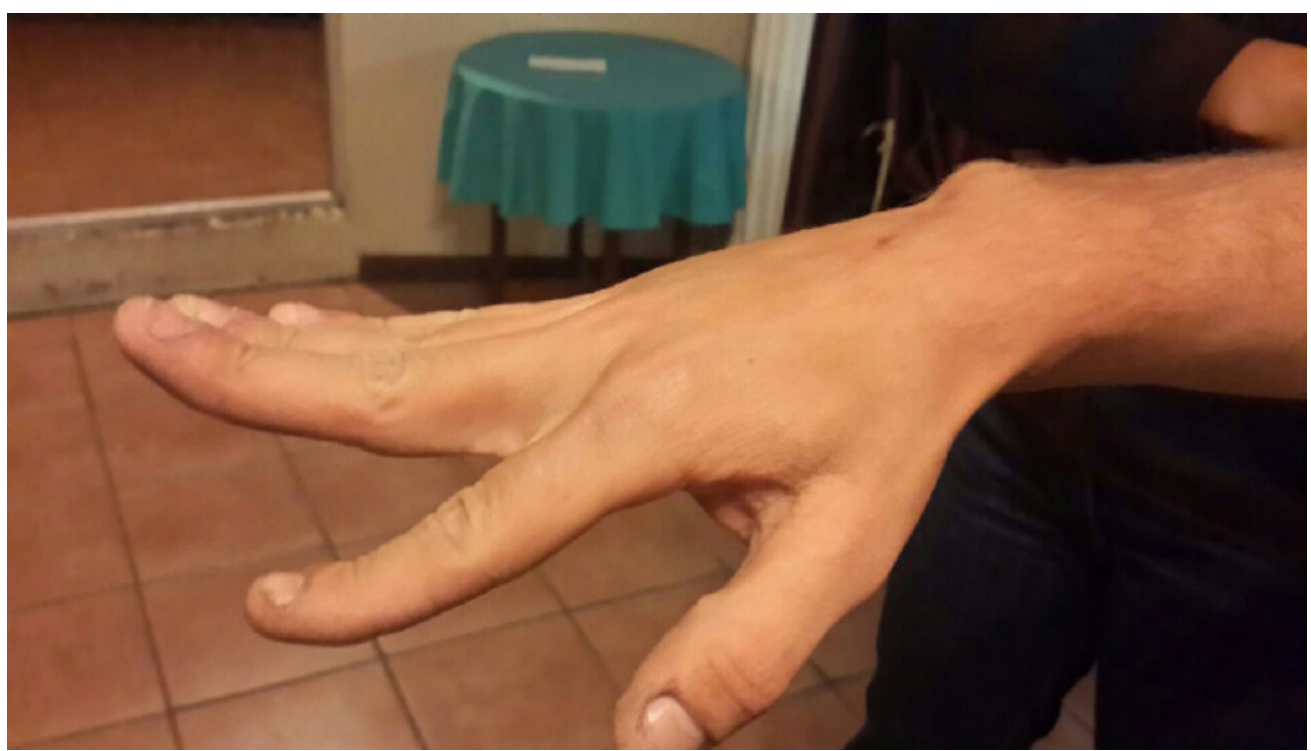

Figure 5: The index finger in neutral clearly showing the extensor tendon

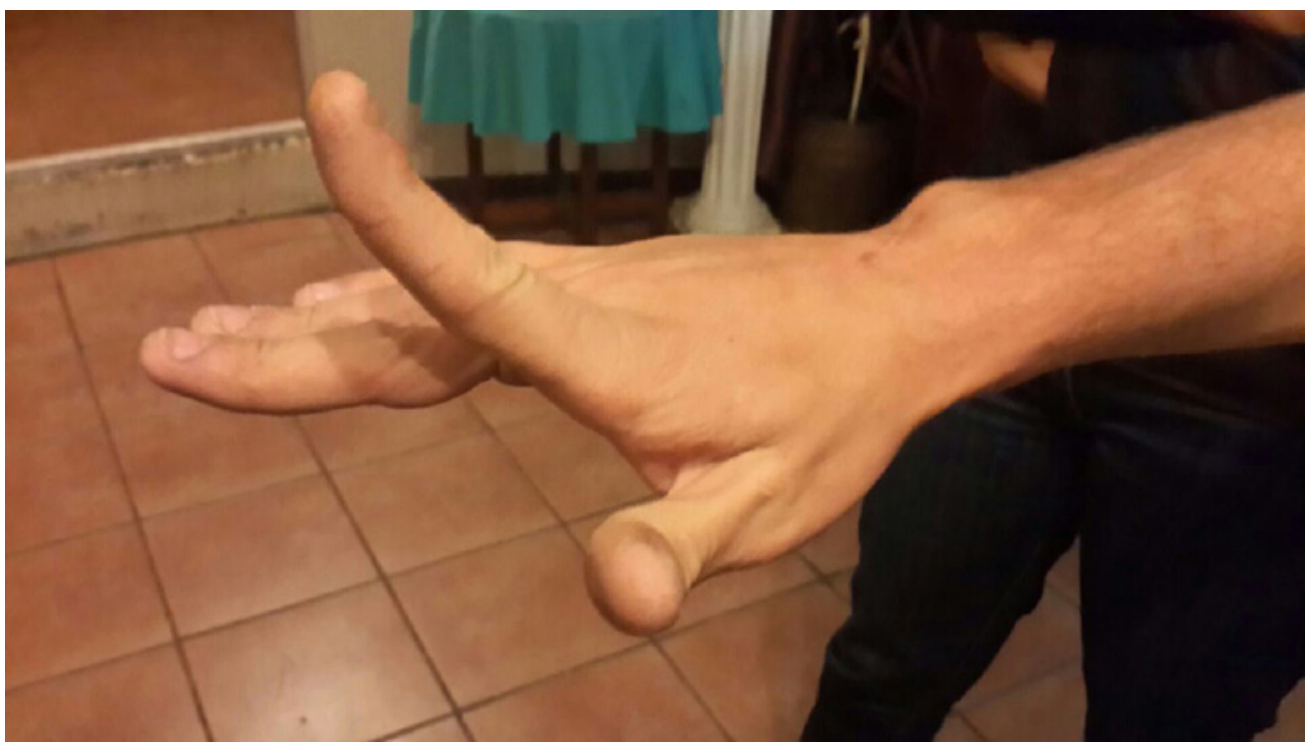

Figure 6: Independent extension of the index finger

\section{Discussion}

Closed rupture of both extensors of the index finger at the musculotendinous junction is extremely rare and most are work injuries with unclear mechanisms. Takami in1995 [3], Mudgal in 2007 [4] and Kumora in 2010 [6] reported cases where work gloves were caught in running machinery and in the same 1995 series, Takami reported one similar case to ours where the injury occurred to a practicing Gymnast. The consensus however is that there has to be resisted finger movement while the muscle is contracting in such a way that there is a longitudinal force working in opposition to the muscular force [1].

Management of these injuries is tendon transfers using the available adjacent tendons as direct repair is not possible. Several of these tendon transfers have been described with almost all giving satisfactory outcomes. The available donors are the Flexor Carpi Radialis [4], the Middle finger Extensor Digitorium Communis [3,6,7] and the Extensor digiti minimi [4,7]. It is interesting though that Komura cited the variable anatomy of the extensors of the little finger where there is sometimes no EDM, as the reason for inferior results of the EDM transfer in his report [7]. In our case, we chose to use the EDC of the middle finger after suturing the two tendons together partly because it is a simple procedure and we got an excellent result. 


\section{Conclusion}

Closed rupture of both extensors of the ring finger at the musculotendinous junction is very rare. The clinical presentation is almost always delayed and the diagnosis can easily be made by lack of the index finger extension and sometimes a soft tender mobile mass under the extensor retinaculum. Combining the distal tendons and suturing to the middle finger EDC is a viable option and should give good results.

\section{References}

1. Collins J, Ishihara Y, Thoma A (2012) Management of digital tendon avulsion at the musculotendinous junction of the forearm: a systematic review. Hand 7: 134-42.

2. Boyes JH, Wilson JN, Smith JW (1960) Flexor-tendon ruptures in the forearm and hand. J Bone Joint Surg Am 42: 637-46.

3. Stuart PR, Briggs PJ (1993) Closed extensor tendon rupture and distal radial fracture with use of a gymnast's wrist support. Br J Sports Med 27: 92-3.

4. Takami H, Takahashi S, Ando M, Suzuki K (1995) Traumatic rupture of the extensor tendons at the musculotendinous junction. J Hand Surg Am 20: 474-7.

5. Mudgal CS, Mudgal S (2007) Closed traumatic rupture of the extensor digitorium communis and extensor indicis proprius at the musculo-tendinous junction. J Hand Surg Eur 32: 675-6.

6. Sunagawa T, Harada A, Ochi M (2009) Traumatic closed index extensor tendon rupture: a case report. J Hand Surg Eur Vol 34: 554-5.

7. Komura S, Yokoi T, Nonomura H (2011) Traumatic closed index extensor tendon rupture at the musculotendinous junction: a report of two cases. Hand 6: 90-2.

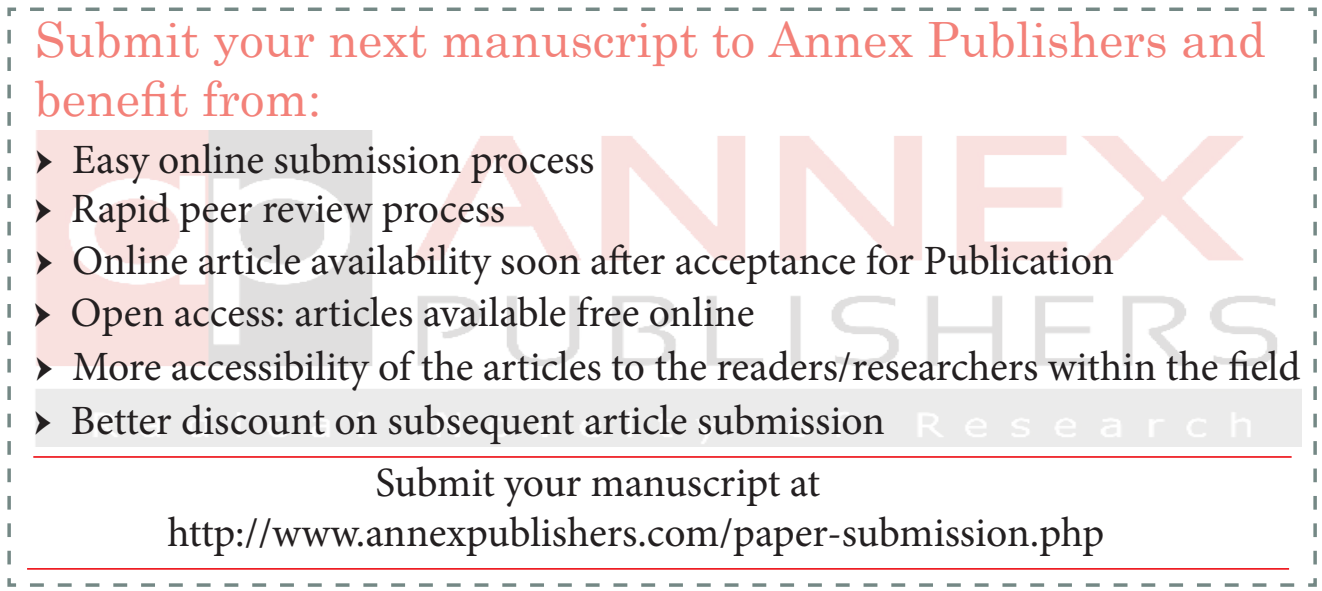

